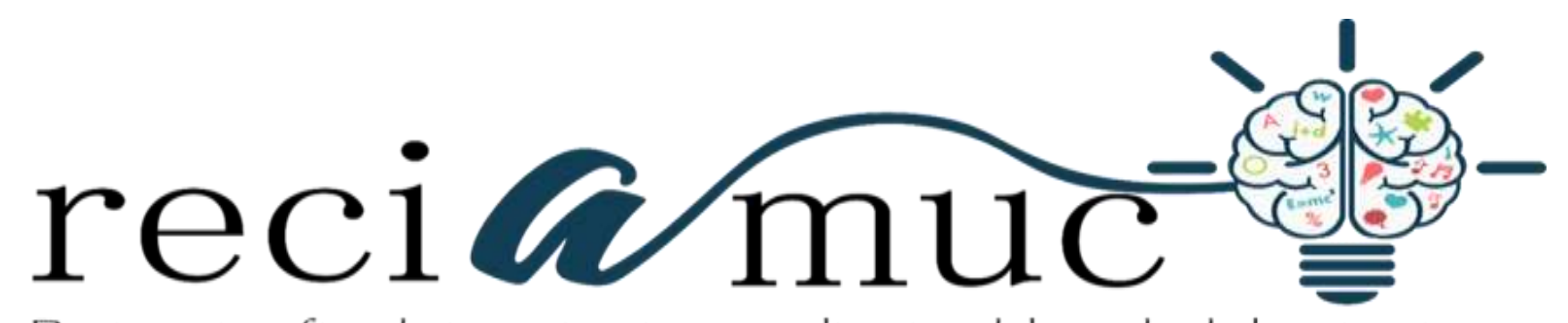

Revista cientifica de investigación actualización del mundo de las ciencias

Eugenio Bolívar Ponce Marcillo a; Cristina Alexandra Vera Moncayo ${ }^{\text {b; }}$ Marlon Alberto Franco Fernández ${ }^{\text {c}}$; Erika Claribel Albán Estrada ${ }^{\mathrm{d}}$

Utilidad laparoscópica diagnóstica en síndrome doloroso abdominal

Diagnostic laparoscopic utility in abdominal pain syndrome

Revista Científica de Investigación actualización del mundo de las Ciencias. Vol. 3 núm., 2, abril, ISSN: 2588-0748, 2018, pp. 933-949

DOI: $10.26820 /$ reciamuc/3.(2).abril.2019.933-949

URL: http://reciamuc.com/index.php/RECIAMUC/article/view/373

Código UNESCO: 3205 Medicina Interna

Tipo de Investigación: Artículo de Revisión

(C) RECIAMUC; Editorial Saberes del Conocimiento, 2019

Recibido: $15 / 01 / 2019$

Aceptado: 07/02/2019

Publicado: 01/04/2019

Correspondencia: samuelaurelio2010@hotmail.com
a. Médico; Investigador Independiente; Guayaquil, Ecuador; samuelaurelio2010@ hotmail.com
b. Médico; Investigador Independiente; Guayaquil, Ecuador; cristy1414@ hotmail.com
c. Médico; Investigador Independiente; Guayaquil, Ecuador; marlon_ff23@ hotmail.com
d. Médico; Investigador Independiente; Guayaquil, Ecuador; ekalaban25@gmail.com 


\section{Utilidad laparoscópica diagnóstica en síndrome doloroso abdominal}

Vol. 3, núm. 2., (2019)

Eugenio Bolívar Ponce Marcillo; Cristina Alexandra Vera Moncayo; Marlon Alberto Franco Fernández; Erika Claribel Albán Estrada

\section{RESUMEN}

El síndrome doloroso abdominal médico corresponde a un diverso conjunto de afecciones de amplias etiologías; su síntoma destacado: el dolor. El dolor se define como una sensación molesta de una parte del cuerpo debida a un estímulo de los centros nerviosos. El síndrome doloroso abdominal agudo no traumático representa un espectro de condiciones benignas y auto limitadas hasta una emergencia quirúrgica. Requiere un enfoque que se base en la probabilidad de enfermedad, la historia del paciente, examen físico, exámenes de laboratorio y estudios de imagen, el enfoque diagnóstico por imagen del síndrome no traumático en adultos va más allá de la regionalización de las cavidades toracoabdominal y pélvica. Estudios por imágenes para evaluar el dolor abdominal basado en la localización del dolor como el ultrasonido se recomienda para evaluar el dolor del cuadrante superior derecho, la tomografía computarizada se sugiere para el dolor en los cuadrantes inferiores. La laparoscópia diagnóstica se emplea para evaluar: el dolor abdominal agudo y crónico, a pacientes críticos con examen abdominal inconcluyente, patologías hepáticas diversas, ascitis, la estatificación de enfermedades neoplásicas malignas intra abdominales, trauma abdominal, procesos de origen ginecológico y en cirugía pediátrica. Algunas contraindicaciones de laparoscópia: inestabilidad hemodinámica, coagulo patía no corregible, severa disfunción cardiorrespiratoria, infección de la pared abdominal, embarazo, entre otras. Algunas emergencias en las cuales el abordaje diagnóstico realizado por laparoscópia es útil: apendicitis aguda, perforación gastroduodenal, la oclusión intestinal y traumatismo abdominal. La laparoscópia diagnóstica evita la realización de estudios preoperatorios innecesarios, retrasos de la intervención quirúrgica, permitiendo una disminución de morbilidad y estadía hospitalaria. La metodología usada es descriptiva, con un enfoque documental, es decir, revisar fuentes disponibles en la red, como google académico, con contenido oportuno y relevante desde el punto de vista científico que enriquezca el análisis del tema planteado en este artículo.

Palabras claves: Síndrome dolor abdominal; Laparoscópia abdominal; Afecciones de amplio espectro; Recuperación más rápida; Efectiva; Mejor visualización. 


\title{
Utilidad laparoscópica diagnóstica en síndrome doloroso abdominal
}

Vol. 3, núm. 2., (2019)

Eugenio Bolívar Ponce Marcillo; Cristina Alexandra Vera Moncayo; Marlon Alberto Franco

Fernández; Erika Claribel Albán Estrada

\begin{abstract}
The abdominal medical painful syndrome corresponds to a diverse of conditions of wide etiologies; its prominent symptom: pain. Pain is defined as an annoying sensation of a part of the body due to a stimulation of the nerve centers. Acute nontraumatic abdominal pain syndrome represents a spectrum of benign and self-limited conditions until a surgical emergency. It requires an approach that is based on the probability of illness, the patient's history, physical examination, laboratory tests and imaging studies, the diagnostic imaging approach of nontraumatic syndrome in adults goes beyond the regionalization of the thoracoabdominal cavities and pelvic Imaging studies to assess abdominal pain based on the location of pain such as ultrasound is recommended to assess the pain of the upper right quadrant, computed tomography is suggested for pain in the lower quadrants. Diagnostic laparoscopy is used to evaluate: acute and chronic abdominal pain, to critically ill patients with inconclusive abdominal examination, various liver pathologies, and ascites, the staging of intra-abdominal malignant neoplastic diseases, abdominal trauma, gynecological processes and pediatric surgery. Some contraindications of laparoscopic: hemodynamic instability, uncorrectable pathological clot, severe cardiorespiratory dysfunction, abdominal wall infection, pregnancy, among others. Some emergencies in which the diagnostic approach performed by laparoscopy is useful: acute appendicitis, gastroduodenal perforation, intestinal occlusion and abdominal trauma. Diagnostic laparoscopy avoids unnecessary preoperative studies, delays in surgical intervention, allowing a decrease in morbidity and hospital stay. The methodology used is descriptive, with a documentary approach that is, reviewing sources available on the web, such as google scholar, with timely and scientifically relevant content that enriches the analysis of the topic raised in this article.
\end{abstract}

Key words: Abdominal pain syndrome; Abdominal laparoscopic; Broad-spectrum conditions; Faster; More effective recovery; Better visualization. 


\section{Utilidad laparoscópica diagnóstica en síndrome doloroso abdominal}

Vol. 3, núm. 2., (2019)

Eugenio Bolívar Ponce Marcillo; Cristina Alexandra Vera Moncayo; Marlon Alberto Franco Fernández; Erika Claribel Albán Estrada

\section{Introducción.}

Las consultas por cuadros de dolor abdominal agudos son comunes en los servicios de urgencia, siendo el dolor abdominal un motivo de consulta habitual. El principal desafío es diferenciar si es un cuadro de resolución quirúrgica o no para el médico tratante. Cuando la vida del paciente depende del tiempo para precisar el diagnóstico es conveniente y necesario hacer uso de todos los medios tecnológicos que garanticen el tratamiento oportuno y alivien el padecimiento del paciente.

Desde que la laparoscopía se introdujo en el campo de la cirugía general, muchos cirujanos la han empleado para el diagnóstico y tratamiento de pacientes con dolor de abdomen agudo, con particular interés cuando no está clara la etiología del cuadro clínico. La laparoscopía diagnóstica se realiza bajo anestesia general, su precisión diagnóstica en pacientes con dolor abdominal agudo es alta, oscila entre $90 \%$ y $100 \%$ y evita laparotomías innecesarias (Sosa, 2019). Se emplea para evaluar: el dolor abdominal agudo y crónico, a pacientes críticos con examen abdominal inconcluyente, patologías hepáticas diversas, ascitis, la estatificación de enfermedades neoplásicas malignas intra abdominales, trauma abdominal, procesos de origen ginecológico y en cirugía pediátrica.

Una laparoscopia diagnóstica puede evitar la necesidad de hacer una operación más grande, innecesaria e invasiva en el futuro, los avances tecnológicos incluida la robótica han permitido una mayor eficiencia y efectividad. El sistema robótico consta de un carrito para el paciente que aloja tres o cuatro brazos y la consola del cirujano, con la que éste maneja dichos brazos. El acceso a la cavidad abdominal se obtiene con las técnicas tradicionales. Una vez colocados los trocares 


\section{Utilidad laparoscópica diagnóstica en síndrome doloroso abdominal}

Vol. 3, núm. 2., (2019)

Eugenio Bolívar Ponce Marcillo; Cristina Alexandra Vera Moncayo; Marlon Alberto Franco Fernández; Erika Claribel Albán Estrada

robóticos, se acoplan los brazos, el cirujano abandona el campo quirúrgico para controlar la consola. Las ventajas del sistema robótico sobre la laparoscopia convencional comprenden una mejora de la óptica y del manejo instrumental.

Desde la consola, el cirujano dispone de una visión tridimensional de la cavidad abdominal, que se logra a través del sistema óptico de dos cámaras (derecha e izquierda) que fusionan las imágenes para dar una visión tridimensional. El procesador del ordenador también puede ampliar las imágenes con más precisión. Es cierto que estas mejoras tecnológicas y en un principio por sus altos costes no estarán disponible para todos los centros, en un futuro cercano se convertirán en la mejor herramienta de diagnóstico del futuro. A continuación, se abordarán las complicaciones y ventajas de laparoscópia diagnostica para el síndrome doloroso abdominal.

\section{Metodología}

Esta investigación está dirigida al estudio del "Utilidad laparoscópica diagnóstica en síndrome doloroso abdominal". Para realizarlo se usó una metodología tipo descriptiva, con un enfoque documental, es decir, revisar fuentes disponibles en la red, como google académico, con contenido oportuno y relevante desde el punto de vista científico para dar respuesta a lo tratado en el presente artículo y que sirvan de inspiración para realizar otros proyectos. Las mismas pueden ser consultadas al final, en la bibliografía.

\section{Resultados}

El síndrome doloroso abdominal médico corresponde al más variado conjunto de afecciones de diversas etiologías; se presentan como síntoma destacado el dolor. Podemos definir 


\section{Utilidad laparoscópica diagnóstica en síndrome doloroso abdominal}

Vol. 3, núm. 2., (2019)

Eugenio Bolívar Ponce Marcillo; Cristina Alexandra Vera Moncayo; Marlon Alberto Franco Fernández; Erika Claribel Albán Estrada

el dolor como una sensación molesta o angustiosa de una parte del cuerpo debida a un estímulo de los centros nerviosos (Patiño, 2017). El síndrome doloroso abdominal agudo no traumático puede representar a un espectro de condiciones benignas y auto limitadas hasta una emergencia quirúrgica. Se presenta en el departamento de urgencias con un amplio espectro de entidades clínicas que lo condicionan, con presentaciones muy variadas lo cual continúa confundiendo a los médicos.

En una larga serie $100 \%$ de los pacientes con apendicitis, colecistitis y obstrucción intestinal refieren dolor seguido de vómitos en contraste con un 20-40\% con síndrome doloroso abdominal agudo inespecífico y gastroenteritis. El síndrome febril y los vómitos son más frecuentes con la apendicitis aguda que en otras causas del síndrome doloroso abdominal agudo (Patiño, 2017).

La evaluación del síndrome doloroso abdominal agudo requiere un enfoque que se base en la probabilidad de enfermedad, la historia del paciente, examen físico, exámenes de laboratorio y estudios de imagen, el enfoque diagnóstico por imagen del síndrome no traumático en adultos va más allá de la regionalización de las cavidades toracoabdominal y pélvica. La ubicación del dolor abdominal en un área específica, en el hemiabdomen superior o inferior o en un cuadrante puede orientar a la patología que lo condiciona.

El uso de la imagen seccional permite evaluar como un todo la cavidad abdominal y su contenido. La ubicación del dolor es un punto de partida útil, por ejemplo, síndrome doloroso abdominal agudo derecho sugiere apendicitis. Algunos elementos de la historia y examen físico 


\section{Utilidad laparoscópica diagnóstica en síndrome doloroso abdominal}

Vol. 3, núm. 2., (2019)

Eugenio Bolívar Ponce Marcillo; Cristina Alexandra Vera Moncayo; Marlon Alberto Franco Fernández; Erika Claribel Albán Estrada

son útiles (estreñimiento y distensión abdominal sugieren fuertemente obstrucción intestinal, con un rango bajo de precisión diagnóstica y de acuerdo entre observadores).

El Colegio Americano de Radiología (Motta, Julio 2017) ha recomendado diferentes estudios por imágenes para evaluar el dolor abdominal basado en la localización del dolor. El ultrasonido se recomienda para evaluar el dolor del cuadrante superior derecho, la tomografía computarizada se recomienda para el dolor en los cuadrantes inferiores. La Laparoscopia diagnostica se emplea para evaluar: el dolor abdominal agudo y crónico, a pacientes críticos con examen abdominal inconcluyente, patologías hepáticas diversas, ascitis, la estatificación de enfermedades neoplásicas malignas intra abdominales, second look, trauma abdominal, procesos de origen ginecológico y en cirugía pediátrica. También es importante tener en cuenta poblaciones especiales como las mujeres que poseen el riesgo de enfermedades genitourinarias y en los adultos mayores que pueden presentar síntomas atípicos de la enfermedad.

Una de las clasificaciones más aceptadas para el dolor abdominal es la de Bockus, en la cual se señalan tres grupos (Motta, Julio 2017):

- Padecimientos intra abdominales que requieren cirugía inmediata.

- Padecimientos abdominales que no requieren cirugía.

- Padecimientos extra abdominales que simulan abdomen agudo.

Desde el punto de vista clínico, en el manejo del paciente se considera en el diagnóstico el origen y manejo de acuerdo al tiempo de evolución quedando en una de dos categorías: agudo (si su duración es de un mes o menos) y crónico (si ha durado más de un mes). 


\section{Utilidad laparoscópica diagnóstica en síndrome doloroso abdominal}

Vol. 3, núm. 2., (2019)

Eugenio Bolívar Ponce Marcillo; Cristina Alexandra Vera Moncayo; Marlon Alberto Franco Fernández; Erika Claribel Albán Estrada

El síndrome doloroso abdominal agudo requiere tomar una decisión diagnóstica y terapéutica en un tiempo breve. Este síndrome es común ya que abarca aproximadamente $30 \%$ del total de las consultas de urgencias y constituye entre 13 y $40 \%$ de todas las urgencias posiblemente quirúrgicas cuando se trata de un origen inespecífico (Montoro, 2012). Si bien el médico clínico posee una gran capacidad y experiencia ante los cuadros clínicos más catastróficos, estos pueden evolucionar con síntomas y signos que obligan al médico tratante a actuar con la mayor eficiencia posible. El objetivo primordial es establecer un diagnóstico temprano, preciso y establecer rápidamente el tratamiento oportuno.

Laparoscopía diagnóstica es un procedimiento que permite la visualización directa de los órganos intra abdominales, casi toda la superficie peritoneal, parte del hígado, vesícula, estómago, píloro y primera porción del duodeno, bazo, intestino delgado, colon y órganos pélvicos. Permitiendo la exploración de los mismos, otorgándole a este estudio la ventaja de ser el único con capacidad terapéutica (Scelza, 2009). Es un procedimiento seguro, tolerado por la mayoría de los pacientes y puede ser realizado como procedimiento ambulatorio bajo anestesia local o general. La figura $\mathrm{N}^{\circ} 1$ ilustra esta técnica. 


\section{Utilidad laparoscópica diagnóstica en síndrome doloroso abdominal}

Vol. 3, núm. 2., (2019)

Eugenio Bolívar Ponce Marcillo; Cristina Alexandra Vera Moncayo; Marlon Alberto Franco

Fernández; Erika Claribel Albán Estrada

\section{Figura $\mathbf{N}^{\circ} 1$ La Laparoscópia}

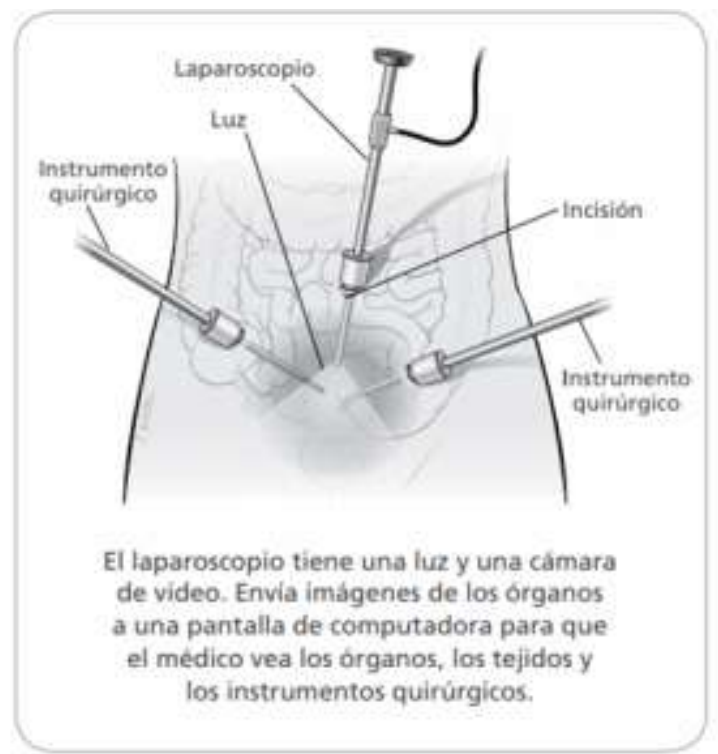

El médico tratante puede recomendar una laparoscopia diagnóstica en los siguientes casos:

- Dolor de larga duración.

- Crecimiento anormal de los tejidos.

- Enfermedad en los órganos del abdomen o la pelvis.

Fuente: (Intermountain Healthcare, 2016)

Mientras se lleva a cabo laparoscopia, el paciente debe estar monitorizado, el lugar debe estar acondicionado con la infraestructura necesaria para maniobras de reanimación o procedimiento quirúrgico. Se realiza bajo estrictas medidas de esterilidad tanto del procedimiento como del equipo laparoscópico. Su sensibilidad oscila entre el 90- $96 \%$, dependiendo de la edad y sexo. En mujeres jóvenes, el porcentaje de error diagnóstico, así como la laparotomía en blanco mediante la cirugía abierta oscila entre 25-50\% (García, 2012).

Contraindicaciones:

- Inestabilidad hemodinámica.

- Paciente que no coopera, íleo paralítico o mecánico.

- Coagulo patía no corregible.

- Enorme hernia hiatal.

- Severa disfunción cardiorrespiratoria. 


\section{Utilidad laparoscópica diagnóstica en síndrome doloroso abdominal}

Vol. 3, núm. 2., (2019)

Eugenio Bolívar Ponce Marcillo; Cristina Alexandra Vera Moncayo; Marlon Alberto Franco Fernández; Erika Claribel Albán Estrada

- Infección de la pared abdominal.

- Multioperado.

- Embarazo.

La decisión final estará determinada por la condición clínica del paciente e informe del cirujano. Este estudio requiere de 20 minutos aproximadamente para su correcta realización, el material básico requiere: un laparoscópico, trocar, pinza de prensión (grasper), retractores, agujas de biopsia para toma de muestras del contenido abdominal. El personal debe incluir al cirujano y un ayudante que se encargue de la monitorización (presión arterial, pulso, frecuencia respiratoria, saturación de oxígeno, ritmo cardiaco y nivel de sedación).

Los pasos que se llevan a cabo la realización de laparoscópia diagnóstica son los siguientes (Intermountain Healthcare, 2016):

1. Se administra anestesia para dormir al paciente. El anestesista le explicará acerca del anestésico que le administrará, cómo funciona y cómo se sentirá el paciente después de la intervención.

2. El cirujano hace unas pequeñas incisiones cerca del ombligo. Las incisiones permiten: introducir un gas inofensivo en el interior del abdomen, este mantiene los órganos separados entre sí, facilitando su observación y trabajar en ellos. Insertar un laparoscópio, tiene una luz y una cámara de video, insertar instrumentos quirúrgicos pequeños para llevar a cabo los procedimientos, colocar un tubo de succión en el abdomen para extraer el exceso de gas o líquido. 


\section{Utilidad laparoscópica diagnóstica en síndrome doloroso abdominal}

Vol. 3, núm. 2., (2019)

Eugenio Bolívar Ponce Marcillo; Cristina Alexandra Vera Moncayo; Marlon Alberto Franco Fernández; Erika Claribel Albán Estrada

3. El médico utiliza el laparoscópio para ver si hay daños o alguna enfermedad. También puede tomar una muestra de tejido (biopsia) que se envía al laboratorio para su análisis, extirpar tejido y/o reparar o extirpar partes de los órganos que estén dañados o enfermos.

4. El laparoscópio y los instrumentos de retiran del cuerpo y el gas se extrae por medio de un tubo de succión. Las incisiones se cierran con suturas (puntos) o grapas.

Debido a su versatilidad diagnóstica y terapéutica que proporciona esta vía de abordaje evita la realización de estudios preoperatorios innecesarios, así como retrasos de la intervención quirúrgica, se traduciría en una disminución de morbilidad y estadía hospitalaria. Las complicaciones pueden ser secundarias a la creación del neumo peritoneo, colocación de los trocares o durante la examinación. También puede presentarse arritmias, inestabilidad hemodinámica por disminución de la precarga, sangrado, fuga biliar, laceración de órganos sólidos, perforación de visera hueca, lesión vascular, embolismo gaseoso, enfisema subcutáneo o extra peritoneal e infección del sitio de acceso, falla en el diagnóstico exacto (DeSimone, 2008).

En años recientes, el dolor abdominal agudo y crónico, las enfermedades neoplásicas del abdomen y el trauma abdominal se han convertido en las indicaciones más frecuentes. La tabla $\mathrm{N}^{\circ} 1$ resume las indicaciones actuales de la laparoscopia diagnostica (Vacuno, 2005).

Tabla 1: Estado actual de la laparoscopia diagnostica

\begin{tabular}{|l|l|}
\hline \multicolumn{1}{|c|}{ Indicaciones electivas } & \multicolumn{1}{|c|}{ Indicaciones de urgencia } \\
\hline Dolor abdominal crónico. & Dolor abdominal agudo o peritonitis. \\
\hline $\begin{array}{l}\text { Patología hepática abdominal penetrante o } \\
\text { cerrado. }\end{array}$ & Casos seleccionados de traumatismo. \\
\hline
\end{tabular}




\section{Utilidad laparoscópica diagnóstica en síndrome doloroso abdominal}

Vol. 3, núm. 2., (2019)

Eugenio Bolívar Ponce Marcillo; Cristina Alexandra Vera Moncayo; Marlon Alberto Franco Fernández; Erika Claribel Albán Estrada

\begin{tabular}{|l|l|}
\hline Ascitis. & $\begin{array}{l}\text { Excluir gangrena intestinal en pacientes } \\
\text { graves. }\end{array}$ \\
\hline $\begin{array}{l}\text { Diagnóstico y estatificación de enfermedades } \\
\text { malignas. }\end{array}$ & \\
\hline $\begin{array}{l}\text { Evaluar la respuesta de diferentes tumores a la } \\
\text { quimioterapia o radioterapia. }\end{array}$ & \\
\hline $\begin{array}{l}\text { Second-look en pacientes con infarto } \\
\text { mesenterico o enfermedades neoplásicas. }\end{array}$ & \\
\hline
\end{tabular}

Fuente: (Vacuno, 2005)

Algunas emergencias en las cuales el abordaje diagnóstico realizado por laparoscópia es útil se mencionan:

Apendicitis aguda: la apendicetomía laparoscópica es un procedimiento relativamente sencillo por esta técnica que no está exento de riesgo de complicaciones. En relación a la apendicectomía por incisión: más tiempo quirúrgico, igualdad de costos, menor tasa de infección de herida, acortamiento en un día de la estancia hospitalaria y recuperación postoperatoria más rápida. La indicación de abordaje laparoscópico en la sospecha de apendicitis debe ser selectiva para el grupo de pacientes que más se benefician.

Perforación gastroduodenal: la incidencia de perforación de ulcus duodenal con peritonitis ha cambiado poco, el ulcus duodenal perforado puede ser eficientemente abordado por el procedimiento laparoscópico. No ocurre lo mismo con la hemorragia digestiva gastroduodenal o la estenosis pilórica, no hay lugar para la laparoscopia de urgencia. El objetivo del abordaje laparoscópico por perforación gastroduodenal debería evitar totalmente la laparotomía, logrando 


\section{Utilidad laparoscópica diagnóstica en síndrome doloroso abdominal}

Vol. 3, núm. 2., (2019)

Eugenio Bolívar Ponce Marcillo; Cristina Alexandra Vera Moncayo; Marlon Alberto Franco Fernández; Erika Claribel Albán Estrada

disminuir el dolor, elimina la posibilidad de infección postoperatoria y eventración, disminuye las complicaciones respiratorias y acorta la baja laboral.

La oclusión intestinal: es la única indicación de laparoscopia que destaca como dificultad la falta de espacio en la cavidad abdominal. Se recomienda en general intervenir de una forma precoz, antes de una gran distensión (inflamación y/o isquemia) de la mayoría del paquete intestinal, que aumentará el riesgo de perforación y dificultará seriamente la creación del espacio de trabajo necesario. Los pacientes multi operados y con abdomen a tensión deben considerarse una contraindicación. La perforación obliga a la conversión inmediata a laparotomía para evitar una peritonitis intra operatoria. Otro argumento para la conversión es el daño de la pared intestinal, esta situación requiere sutura manual.

Traumatismo abdominal: cualquier traumatismo abdominal podría ser tratable por laparoscopia, las causas más frecuentes son el hemoperitoneo pequeño por trauma abdominal cerrado (originado en lesiones superficiales de hígado o bazo) y la perforación de víscera hueca por trauma penetrante, también pueden ser diagnosticadas y en ocasiones tratadas con abordaje laparoscópico. Otras lesiones abdominales (hematoma retroperitoneal o de mesorotura diafragmática, rotura vesical). El objetivo fundamental de la laparoscopia reside en evitar la laparotomía exploradora sin ningún gesto quirúrgico (porque se ha realizado hemostasia espontánea del hemoperitoneo o se trata de un hematoma retroperitoneal), y la laparotomía sólo para hacer un gesto hemostático menor (principalmente sobre erosiones superficiales en el hígado y en el bazo). 


\section{Utilidad laparoscópica diagnóstica en síndrome doloroso abdominal}

Vol. 3, núm. 2., (2019)

Eugenio Bolívar Ponce Marcillo; Cristina Alexandra Vera Moncayo; Marlon Alberto Franco Fernández; Erika Claribel Albán Estrada

El propósito de la laparoscopia en el traumatismo abdominal es fundamentalmente diagnóstico (laparoscopia exploradora, que evite una laparotomía en blanco) y marginalmente terapéutica. Aumenta la seguridad diagnóstica en casos clínicamente y radiológicamente dudosos de ser susceptibles de observación quirúrgica; si no hay lesiones pueden ser dados de alta de forma temprana, con mayor seguridad.

Teniendo en consideración la gravedad del paciente politraumatizado, se debe fijar un umbral bajo de conversión a laparotomía en algunas situaciones: hemorragia masiva y hemorragia no controlable por laparoscopia, heridas profundas (mayor de $3 \mathrm{~cm}$ ) en hígado o bazo, no localización de la lesión sangrante y hematoma sub capsular esplénico expansivo o mayor de 1/3 de su superficie (Balén, 2005).

Algunas de las contraindicaciones de este procedimiento:

- Inestabilidad hemodinámica: reviste una gravedad mayor que en otras urgencias abdominales.

- Traumatismo torácico severo: por las dificultades de ventilación mecánica y de intercambio gaseoso que ya de por sí produce la laparoscopia (añadidas al trauma torácico).

- Traumatismo craneal no estudiado: por el riesgo de que la hiperpresión abdominal aumente la presión intracraneal, al transmitir la presión intrabdominal al drenaje venoso de la yugular interna a través del sistema cava.

- Sospecha de rotura del diafragma: en ese caso debe colocarse un drenaje torácico antes de comenzar la laparoscopia para evitar un neumotórax a tensión al realizar el neumoperitoneo, si efectivamente hay rotura diafragmática. 


\section{Utilidad laparoscópica diagnóstica en síndrome doloroso abdominal}

Vol. 3, núm. 2., (2019)

Eugenio Bolívar Ponce Marcillo; Cristina Alexandra Vera Moncayo; Marlon Alberto Franco

Fernández; Erika Claribel Albán Estrada

Riesgos del uso de la Laparoscópia en trauma

Ante la presencia de heridas diafragmáticas existe la probabilidad de generar neumotórax a tensión, o neumo pericardio en la realización de técnica laparoscópia en trauma. Teóricamente es así; sin embargo, en la práctica ante la sospecha de lesión diafragmática, la colocación profiláctica de drenaje torácico disminuye la posibilidad que se presente esta complicación.

La complicación más temida en el empleo de la laparoscopia en trauma es la falta de reconocimiento de lesiones; (lesiones desapercibidas). La experiencia del cirujano, su habilidad técnica, disposición del equipo y material necesario para la correcta realización del procedimiento. No se debe olvidar que ante la duda es siempre conveniente convertir a una laparotomía (Rodríguez, 2018).

Otra de las complicaciones latente es la imposibilidad de repetir cada uno de los pasos quirúrgicos realizados mediante el abordaje convencional, una adecuada revisión de la cavidad abdominal y evacuación de restos hemáticos que pudieran condicionar padecimiento intrabdominal e infección.

\section{Conclusión.}

La laparoscópia diagnóstica constituye una técnica eficiente, efectiva y útil al tratar el síndrome de dolor abdominal porque permite precisar la causa de la molestia del paciente cuando la causa o etiología no está del todo clara o precisa. Esto no significa que el doctor no quiera evaluar al paciente con métodos tradicionales, sino que la tecnología llego al área de la medicina para quedarse. 


\section{Utilidad laparoscópica diagnóstica en síndrome doloroso abdominal}

Vol. 3, núm. 2., (2019)

Eugenio Bolívar Ponce Marcillo; Cristina Alexandra Vera Moncayo; Marlon Alberto Franco Fernández; Erika Claribel Albán Estrada

La laparoscopia es una técnica que debe aprenderse, exige una serie especial de pericias y requisitos que no necesariamente se pueden trasladar a un cirujano veterano formado antes de la llegada de esta técnica. La nueva instrumentación, las dificultades de exposición, la dependencia de la electrocirugía y la retroacción táctil nominal requieren unos conocimientos quirúrgicos diferentes. Durante los $\mathrm{u}^{\prime}$ últimos años ha quedado claro que la laparoscopia se puede aplicar perfectamente a las mujeres, hombres, niños y ancianos. Muchas intervenciones se realizan con menos perdidas hemáticas, menos complicaciones de la herida y estancias hospitalarias más cortas.

Algunos retractores de la práctica de la laparoscópia diagnóstica sin permitir la posibilidad de realizar, en el mismo acto quirúrgico, procedimientos operatorios, causa incrementos injustificados y excesivos en los costos de la atención de las pacientes. Esto unido a la frecuencia de patología encontrada en pacientes con síndrome dolor abdominal implica que todas las laparoscopias diagnósticas realizadas a pacientes deban permitir realizar procedimientos intra operatorios por laparoscopia. Esta posibilidad debe discutirse ampliamente con el paciente antes de realizar cualquier procedimiento diagnóstico y/u operatorio. Otros estudios relevan que, si bien es más costoso que la técnica tradicional, existen otros costos asociados a laparoscópia como la recuperación más expedita del paciente, una menor estancia hospitalaria, entre otras.

La complicación más temida en el empleo de la laparoscopia en trauma es la falta de reconocimiento. La experiencia del cirujano, su habilidad técnica, disposición del equipo y material necesario para la correcta realización del procedimiento. Otra de las complicaciones latente es la imposibilidad de repetir cada uno de los pasos quirúrgicos realizados mediante el 


\section{Utilidad laparoscópica diagnóstica en síndrome doloroso abdominal}

Vol. 3, núm. 2., (2019)

Eugenio Bolívar Ponce Marcillo; Cristina Alexandra Vera Moncayo; Marlon Alberto Franco

Fernández; Erika Claribel Albán Estrada

abordaje convencional, una adecuada revisión de la cavidad abdominal y evacuación de restos hemáticos que pudieran condicionar padecimiento intrabdominal e infección.

\section{Bibliografía}

Balén, E. (2005). El papel de la laparoscopia en la cirugía abdominal urgente. Revista Anales del Sistema Sanitario de Navarra; 28(3).

DeSimone, C. (2008). Laparoscopia ginecológica. Revista Clínica Quirúrgica de Norteamérica; 88(1): 319-341.

García, G. (abril de 2012). Laparotomía y/o laparoscópia diagnóstica en abdomen agudo no traumático en adultos. Obtenido de Instituto Mejicano del Seguro Social IMSS: www.ceneted.salud,gob.mx

Intermountain Healthcare. (12 de diciembre de 2016). Laparoscopia diagnóstica. Obtenido de Intermountain Healthcare: www.intermountainhealthcare.org

Montoro, M. A. (agosto de 2012). Dolor abdominal agudo. Obtenido de ELSEVIER: www.elsevier.es

Motta, G. (Julio 2017). El enfoque diagnóstico por imagen del síndrome doloroso abdominal agudo no traumático en adultos. Revista Anales de Radiología México;16(3) 147-177.

Patiño, F. (2017). Síndrome Doloroso Abdominal. Aspectos Médicos. Revista chilena de pediatría. Volumen $28 \mathrm{~N}^{\circ} 2,110-111$, recuperado por http: www.scielo.conicyt.cl.

Rodríguez, C. (10 de diciembre de 2018). La importancia de la laparoscopia en trauma y cirugías de urgencia. Obtenido de Librería Médica Distribuna: www.libreriamedica.com

Scelza, A. (marzo de 2009). Laparoscopia en el Abdomen Agudo. Obtenido de MEDNET: www.mednet.org,uy

Sosa, A. (2019). Análisis de la laparoscopía diagnóstica y. Revista Médica de Uruguay Volumen $35 \mathrm{~N}^{\circ}$ (1), 27-35, recuperado por http: www.rmu.org.uy.

Vacuno, D. (2005). Laparoscopía Diagnóstica. Revista de Cirugía Paraguaya. Vol. 28; №1, 64-73, recuperado por http: www.sopaci.org.py.

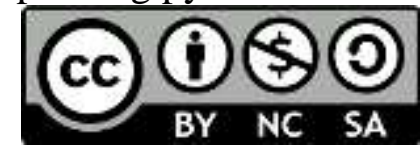

RECONOCIMIENTO-NOCOMERCIAL-COMPARTIRIGUAL

CC BY-NC-SA

ESTA LICENCIA PERMITE A OTROS ENTREMEZCLAR, AJUSTAR Y CONSTRUIR A PARTIR DE SU OBRA CON FINES NO COMERCIALES, SIEMPRE Y CUANDO LE RECONOZCAN LA AUTORÍA Y SUS NUEVAS CREACIONES ESTÉN BAJO UNA LICENCIA CON LOS MISMOS TÉRMINOS. 